\title{
Phase shift cavity ring down absorption spectroscopy
}

\author{
Richard Engeln, Gert von Helden, Giel Berden, Gerard Meijer \\ Departent of Molecular and Laser Physics, University of Nijmegen, Toernooiveld 1, 6525 ED Nijmegen, The Netherlands
}

Received 14 June 1996

\begin{abstract}
Cavity ring down absorption spectroscopy with a continuous light source is used to measure the transition frequencies and absolute absorption coefficient of the weak $\mathrm{b}^{1} \Sigma_{\mathrm{g}}^{+}\left(v^{\prime}=2\right) \leftarrow \mathrm{X}^{3} \mathrm{\Sigma}_{\mathrm{g}}^{-}\left(v^{\prime \prime}=0\right)$ transition of ${ }^{18} \mathrm{O}_{2}$. The absorption spectrum is extracted from a measurement of the magnitude of the phase shift that an intensity modulated continuous light beam experiences upon passing through an unstabilized optical cavity.
\end{abstract}

\section{Introduction}

Application of direct absorption techniques is advantageous in a variety of research fields, since quantitative concentration data as well as absolute frequency dependent absorption cross-sections can be extracted from the measurements. For these reasons, sensitive absorption spectroscopic techniques have gained renewed interest, even in areas where more sophisticated laser-based diagnostic techniques are commonly applied. Among the variety of techniques that have been explored, the cavity ring down (CRD) absorption technique has proven to be a valuable addition as it combines a good detection sensitivity with a rather simple and straightforward experimental setup. The CRD absorption technique is based on the measurement of the photon lifetime in an optical cavity after excitation with a light source and is independent of light source intensity fluctuations. Use of CRD for sensitive absorption measurements with pulsed lasers was first demonstrated by O'Keefe and Deacon in 1988 [1]. Since then this technique has been used for absorption measurements on molecules in pulsed molecular beams [2-5], for trace gas de- tection of molecules in ambient air and/or in hostile environments [6-8] and for the measurement of absolute oscillator strengths of weak transitions of stable molecules $[9,10]$, to just name a few. The CRD technique has been applied over the $200-3500 \mathrm{~nm}$ range $[8,11]$ and a broadband version of this technique, that is promising for exploration in the spectroscopically valuable IR region of the spectrum, has recently been demonstrated [12].

Encouraged by its success, various schemes of CRD absorption spectroscopy are currently under consideration. In particular, for absorption measurements on static samples one would like to improve on the duty cycle; in a typical CRD experiment with a $10-100$ $\mathrm{Hz}$ repetition frequency laser and cavities with photon lifetimes of around 1-10 $\mu \mathrm{s}$, one effectively measures only during a small fraction of the time. At this point the work reported by Herbelin et al. [13] and Anderson et al. [14], work that is to be regarded as a precursor to the CRD technique, is worth to be brought back into notice. Herbelin et al. [13] used intensity modulated continuous light beams and phase shift detection to accurately measure the reflectance of mirror coatings, whereas Anderson demonstrated that similar 
measurements could be performed by abruptly switching off the continuous light source and recording the subsequent intensity decay of the light in the optical cavity. Both methods can be applied for sensitive absorption measurements on gas phase samples inside the cavity as well, and have the additional advantage that the light source can be used more efficiently as the intensity inside the cavity is allowed to be build up during a prolonged time-period. The use of $\mathrm{cw}$ light sources in CRD absorption detection will greatly increase the capability of this technique. Not only will it enable sensitive high resolution studies but it is also expected to lead to dedicated low cost absorption detectors.

In this Letter we report on the use of a tunable narrowband continuous laser for sensitive CRD absorption measurements on the weak $\mathbf{b}^{1} \Sigma_{\mathbf{g}}^{+}\left(v^{\prime}=2\right) \leftarrow$ $\mathrm{X}^{3} \Sigma_{\mathrm{g}}^{-}\left(v^{\prime \prime}=0\right)$ band of ${ }^{18} \mathrm{O}_{2}$. The absorption spectrum is extracted from a measurement of the wavelength dependent phase shift (PS) that an intensity modulated $\mathrm{cw}$ light beam experiences upon passing through a high $Q$ optical cavity. As the PS-CRD measurement method is independent of random light source intensity fluctuations, it can be performed in a cavity without any stabilization of the cavity length, as will be explicitly demonstrated. A comparison of the PS-CRD technique relative to the CRD technique using tunable pulsed light sources is given.

\section{The experimental method}

The light that is used for the experiment is obtained from an $\mathrm{Ar}^{+}$laser pumped single-mode $\mathrm{cw}$ ring dye laser (Spectra Physics 380), operating on DCM. The effective bandwidth of the laser is about $1 \mathrm{MHz}$. The output of the laser is attenuated to about $1 \mathrm{~mW}$ and is passed through an electro-optical modulator (EOM) that sinusoidally modulates the light intensity. The intensity modulated light beam is directed toward the optical cavity, formed by two identical highly reflecting plano-concave mirrors. To be able to couple the laser beam into the optical cavity, two approaches are possible. The commonly used, but also most involved, approach is to make a confocal cavity, i.e. to choose the distance $d$ between the mirrors identical to the radius of curvature $r$. A confocal cavity has a sparse but well-defined mode structure and light can be coupled into the cavity only when the wavelength of the light is resonant with one of the modes. In practice, therefore, the length of the cavity has to be actively locked to the wavelength of the laser, and has to track the laser wavelength while scanning. The other approach is to make a so-called stable optical cavity, and choose the mirror separation $d$ to be within the stability regime given by $0<d<r, r<d<2 r$. The mode structure of such a cavity can be made very dense, especially when the $d / r$ ratio is chosen to be an irrational number. Even though each of the individual cavity modes is very narrow, as determined by the mirror reflectivity, there is a sufficient number of modes that the narrowband $\mathrm{cw}$ laser beam will be coupled into the cavity from time to time. The overall efficiency with which the laser light is coupled into the cavity is less than in the confocal setup and will strongly fluctuate in time as it depends on the amount of cavity length (de) stabilization.

In our experimental setup we choose the second approach, and the cavity we used is formed by a stainless steel tube, $45 \mathrm{~cm}$ long and with an inner diameter of $8 \mathrm{~mm}$, closed on both sides by two highly reflecting mirrors. The mirrors have a diameter of $1^{\prime \prime}$, a radius of curvature of $r=-25 \mathrm{~cm}$ and a reflectivity of (only) $R=0.9985$ around $635 \mathrm{~nm}$. The light that leaks out of the cavity is detected by a photo multiplier tube (PMT) placed closely behind the cavity in order to ensure that all cavity modes are detected with equal probability. In Fig. 1 the time-dependence of the light intensity behind the cavity, as measured with the PMT and displayed on a digital oscilloscope, is shown. Over a $30 \mu$ s time-interval the stabilized narrowband laser is seen to be coupled into the unstabilized optical cavity several times, each time for typically a fraction of a $\mu$ s. On the average, the light is seen to be coupled efficiently into the cavity about $5 \%$ of the time. Superimposed on the transmission maxima of the cavity the high frequency intensity modulation imposed by the electro-optical modulator can be recognized.

The time dependence of the light intensity that enters the optical cavity $I_{\text {exc }}(t)$ can be written in terms of the modulation depth $\alpha$ and the angular modulation frequency $\Omega$ as

$I_{\text {exc }}(t)=I_{0}(1+\alpha \sin \Omega t)$.

It was already shown in 1933 [15] that if this intensity modulated light beam were to excite an atomic 


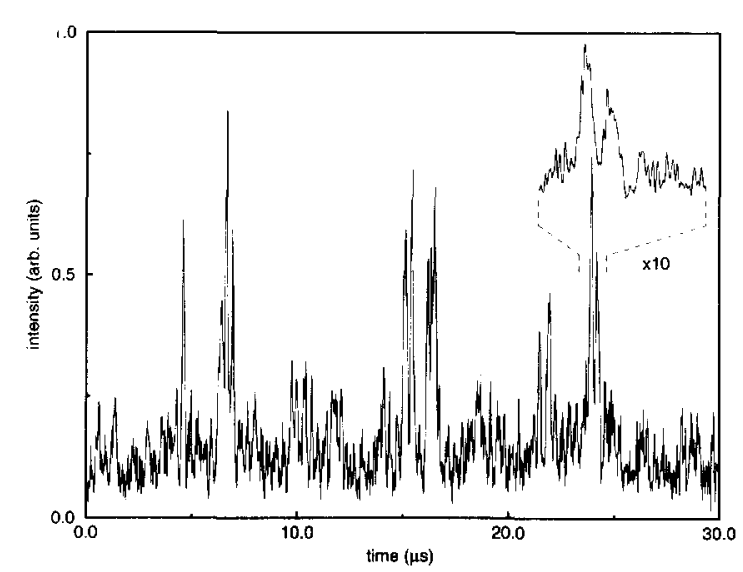

Fig. 1. The time-dependence of the light intensity behind the unstabilized optical cavity, as recorded with a PMT connected to a digital oscilloscope.

or molecular energy level, the resulting fluorescence would also be modulated in time with angular frequency $\Omega$, but with a phase shift relative to the phase of the exciting beam. The phase shift $\phi$ can be related to the lifetime $\tau$ of the excited state via

$\tan \phi=-\Omega \tau$

The finite lifetime $\tau$ also influences the modulation depth which decreases by a factor

$\frac{1}{\sqrt{1+\Omega^{2} \tau^{2}}}$

Already in the mid-fifties lifetimes of fluorescing substances in the ns range were measured via this phase shift method with $1 \%$ or better precision $[16,17]$. Nowadays the phase shift method is still in use to distinguish species on the basis of their lifetime in twodimensional fluorescence imaging [18].

In a PS-CRD experiment the system to be excited with the intensity modulated light beam is the stable optical cavity. The light intensity inside the cavity also decays exponentially in time, and the frequency dependent 1 /e photon lifetime $\tau(\nu)$ of the optical cavity can be written as

$\tau(\nu)=\frac{d}{c\left[\left|\ln \left(R_{\mathrm{eff}}\right)\right|+\sum_{i} \sigma_{i}(\nu) \int_{0}^{d} n_{i}(x) \mathrm{d} x\right]}$.

Here $d$ is the length of the cavity, $c$ is the speed of light, $R_{\text {eff }}$ is the effective reflectivity of the mirrors and the sum is over all light scattering and absorbing species with frequency dependent cross-sections $\sigma_{i}(\nu)$ and a line-integrated number density $\int_{0}^{d} n_{i}(x) \mathrm{d} x$. If only one absorbing species is homogeneously distributed throughout the optical cavity the sum can be rewritten as $\sigma(\nu) n d=\kappa(\nu) d$, where the absorption coefficient $\kappa(\nu)$ is the quantity to be determined. In the case of an intensity modulated $\mathrm{cw}$ light source the intensity measured behind the cavity at a time $t, I_{\mathrm{CRD}}(t)$, is given by

$$
\begin{aligned}
I_{\mathrm{CRD}}(t) & \frac{1}{\tau(\nu)} \int_{-\infty}^{t} I_{0}\left(1+\alpha \sin \Omega t^{\prime}\right) \exp \left[-\frac{\left(t-t^{\prime}\right)}{\tau(\nu)}\right] \mathrm{d} t^{\prime} \\
= & I_{0}\left(1+\frac{\alpha}{1+\Omega^{2} \tau(\nu)^{2}}[\sin \Omega t-\Omega \tau(\nu) \cos \Omega t]\right) \\
= & I_{0}\left(1+\frac{\alpha}{\sqrt{1+\Omega^{2} \tau(\nu)^{2}}}\right. \\
& \times \sin [\Omega t-\arctan \Omega \tau(\nu)])
\end{aligned}
$$

It follows, therefore, that the 'cavity ring down' time $\tau(\nu)$, and thereby the absorption coefficient $\kappa(\nu)$, can be determined in an intensity-independent way from a measurement of the ratio of the in-phase component to the out-of-phase component of the modulated light intensity that exits the optical cavity as a function of laser wavelength. For optimum detection sensitivity, the angular modulation frequency $\Omega$ is selected such that $\Omega \tau(\nu)$ is around 1, i.e. an angular frequency of around $1 \mathrm{MHz}$ is selected for cavities with a ring down time of around $1 \mu \mathrm{s}$.

The signal of the PMT is amplified by a low-noise preamplifier (Stanford Research Systems, SR560) and analysed with a fast lock-in amplifier (EG\&G PAR, model 5202). The voltages on the EOM are set in such a way that the frequency of the generated light intensity modulation is twice the frequency of the driving RF generator, so one can easily discriminate against noise generated by the RF power amplifier. The signal of the RF generator is doubled to obtain a reference frequency for the lock-in amplifier. In order to decrease crosstalk of the RF generating circuit to the lock-in amplifier, they are electronically decoupled by means of an opto-isolator. The lock-in amplifier generates three output signals, an in-phase 
signal, an out-of-phase signal and the phase-angle, which are all three recorded during a scan. In addition, the $\mathrm{I}_{2}$ absorption spectrum is recorded for absolute frequency calibration.

\section{Results and discussion}

As the total volume of our PS-CRD absorption cell is only $25 \mathrm{~cm}^{3}$, direct absorption studies of weak transitions of rather expensive rare isotopes can be afforded. ${ }^{18} \mathrm{O}_{2}$ from a $0.25 \mathrm{~atm} .1$. break-seal flask with a stated purity of $99.2^{18} \mathrm{O}$ atom \% (Campro Scientific) is used without further purification. Measurements are made on the $\mathrm{b}^{1} \Sigma_{\mathrm{g}}^{+}\left(v^{\prime}=2\right) \leftarrow \mathrm{X}^{3} \Sigma_{\mathrm{g}}^{-}\left(v^{\prime \prime}=0\right)$ band of ${ }^{18} \mathrm{O}_{2}$. The electronic transition is a singlet-triplet intercombination band of a magnetic dipole allowed transition and the particular vibrational band has in addition a rather unfavorable Franck-Condon factor. Compared to fully allowed electric dipole transitions, the band under study is more than ten orders of magnitude weaker and therefore a good candidate to demonstrate the capabilities of the PS-CRD method. To the best of our knowledge, this band has not been reported before.

To determine the absolute value of the phase shift imposed by the cavity, the cavity is bypassed, thereby defining the zero phase shift. With the empty cavity aligned, the angular modulation frequency of the intensity is set to $2.13 \mathrm{MHz}$. This results in a phase shift of about $50^{\circ}$, corresponding to a ring down time of 560 ns. The cavity is then filled with ${ }^{18} \mathrm{O}_{2}$, and the phase shift of the light exiting the cavity is measured as a function of laser wavelength. Part of the measured spectrum is shown in Fig. 2. The spectrum is actually constructed from the ratio of the in-phase to the outof-phase component of the modulated intensity exiting the cavity as measured in 85 partly overlapping wavelength scans each over approximately $1 \mathrm{~cm}^{-1}$. The lock-in amplifier is set to integrate over a time of 1 $\mathrm{s}$, corresponding to an integration over approximately $0.002 \mathrm{~cm}^{-1}$ during the wavelength scan. On the vertical scale, the quantity $(c \tau(\nu))^{-1}$ is plotted, which shows the absorption spectrum on an absolute scale, superimposed on a baseline given by $\left(\left|\ln \left(R_{\mathrm{eff}}\right)\right| / d\right)$. The spectrum is recorded with an oxygen pressure of 365 mbar. At this pressure the width of the absorption lines is mainly determined by the Doppler broad-

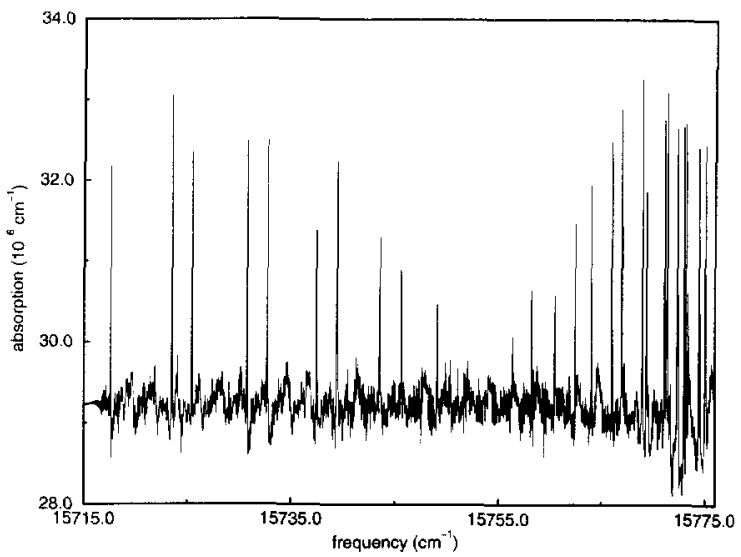

Fig. 2. The absorption spectrum of 365 mbar of ${ }^{18} \mathrm{O}_{2}$ around 635 $\mathrm{nm}$ measured via the PS-CRD method. The spectral structure is due to the $\mathrm{b}^{1} \Sigma_{\mathrm{g}}^{+}\left(v^{\prime}=2\right) \leftarrow \mathrm{X}^{3} \Sigma_{\mathrm{g}}^{-}\left(v^{\prime \prime}=0\right)$ transition.

Table 1

Absolute frequencies (in $\mathrm{cm}^{-1}$ ) and assignments of rotational lines in the $b^{1} \Sigma_{\mathrm{g}}^{+}\left(v^{\prime}=2\right) \leftarrow \mathrm{X}^{3} \Sigma_{\mathrm{g}}^{-}\left(v^{\prime \prime}=0\right)$ band of ${ }^{18} \mathrm{O}_{2}$. The spectroscopic notation ${ }^{\Delta N} \Delta J$ is used, in which $N$ is the end-overend rotational quantum number and $J$ is the quantum number of the total angular momentum, including the electron spin

\begin{tabular}{rllll}
\hline$N^{\prime \prime}$ & ${ }^{P_{P}}$ & ${ }^{P_{Q}}$ & ${ }^{{ }}$ & ${ }^{{ }} \mathrm{Q}$ \\
\hline 1 & $15749.13(3)$ & - & $15756.4(2)$ & $15758.2(2)$ \\
3 & $15743.60(3)$ & $15745.65(3)$ & $15760.46(3)$ & $15762.46(3)$ \\
5 & $15737.48(3)$ & $15739.48(3)$ & $15764.01(5)$ & $15766.0(1)$ \\
7 & $15730.79(3)$ & $15732.77(3)$ & $15766.97(5)$ & $15768.98(5)$ \\
9 & $15723.54(5)$ & $15725.47(3)$ & $15769.35(5)$ & $15771.38(5)$ \\
11 & $15715.69(3)$ & $15717.6(1)$ & $15771.12(5)$ & $15773.18(5)$ \\
13 & $15707.29(3)$ & $15709.19(3)$ & $15772.35(5)$ & $15774.41(3)$ \\
15 & $15698.29(3)$ & $15700.19(3)$ & $15773.0(1)$ & $15775.0(1)$ \\
17 & $15688.74(5)$ & $15690.61(5)$ & $15773.0(1)$ & $15775.1(1)$ \\
19 & $15678.61(5)$ & $15680.46(5)$ & $15772.4(1)$ & $15774.56(3)$ \\
21 & $15667.9(2)$ & $15669.7(2)$ & $15771.3(1)$ & $15773.4(1)$ \\
23 & & & & $15771.70(5)$ \\
\hline
\end{tabular}

ening in the room temperature cell, and a full width at half maximum of $0.045 \mathrm{~cm}^{-1}$ is found for isolated absorption lines. The absolute line positions, together with their spectroscopic identification, are tabulated in Table 1. The presented values are determined for every scan separately, using the $I_{2}$ calibration spectrum [19]. In this way an accuracy of about 0.2 to $0.03 \mathrm{~cm}^{-1}$ is obtained for the absolute frequency of the lines, depending on the number of iodine lines in a scan as well as on the position of the iodine lines relative to the oxygen lines. 
The independence of intensity fluctuations allows the PS-CRD absorption method to be performed in optical cavities without any length stabilization. It is explicitly noted that the reported absorption spectrum is too weak to be measured by recording the total intensity passing through the cavity; in a plot of the sum of the square of the in-phase and the square of the out-of-phase component against wavelength the ${ }^{18} \mathrm{O}_{2}$ absorption features are no longer visible.

\section{Conclusions}

Sensitive absorption detection on gas-phase samples is demonstrated using an intensity modulated continuous laser beam, coupled into an optical cavity. The absorption information is deduced from the wavelength dependent phase shift that the modulated beam undergoes upon passing through the cavity. The spectral resolution of the measurement is in principle only limited by the bandwidth of the laser $(1 \mathrm{MHz}$ in our case) although in the experiment reported here on the $\mathrm{b}^{1} \Sigma_{\mathrm{g}}^{+}\left(v^{\prime}=2\right) \leftarrow \mathrm{X}^{3} \Sigma_{\mathrm{g}}^{-}\left(v^{\prime \prime}=0\right)$ transition of ${ }^{18} \mathrm{O}_{2}$ around $635 \mathrm{~nm}$, Doppler broadening is the limiting factor. The detection sensitivity compares favorable to the detection sensitivity that is obtained in the same experimental setup when a narrowband pulsed laser is used for 'standard' CRD absorption spectroscopy. This can be understood from the greatly improved duty cycle in the experiment when continuous rather than pulsed light sources are used. Further improvement of the detection sensitivity can be achieved by actively locking the laser to the cavity and thus coupling light into the cavity more efficiently. The possibility to use cw light sources and analog detection electronics makes the PS-CRD technique applicable not only in fundamental high resolution spectroscopic studies, but also in trace gas detection systems.

\section{Acknowledgement}

This work is part of the research program of the 'Stichting voor Fundamenteel Onderzoek der Materie
(FOM)', which is financially supported by the 'Nederlandse Organisatie voor Wetenschappelijk Onderzoek (NWO)', and receives direct support by NWO via PIONIER-grant \# 030-66-89.

\section{References}

[1] A. O'Keefe and D.A.G. Deacon, Rev. Sci. Instrum. 59 (1988) 2544.

[2] A. O'Keefe, J.J. Scherer, A.L. Cooksy, R. Sheeks, J. Heath and R.J. Saykally, Chem. Phys. Lett. 172 (1990) 214

[3] J.J. Scherer, J.B. Paul, C.P. Collier and R.J. Saykally, J. Chem. Phys. 102 (1995) 5190.

[4] J.B. Paul, J.J. Scherer, C.P. Collier and R.J. Saykally, J. Chem. Phys. 104 (1996) 2782.

[5] M.G.H. Boogaarts and G. Meijer, J. Chem. Phys. 103 (1995) 5269.

[6] G. Meijer, M.G.H. Boogaarts, R.T. Jongma, D.H. Parker and A.M. Wodtke, Chem. Phys. Lett. 217 (1994) 112.

[7] P. Zalicki, Y. Ma, R.N. Zare, E.H. Wahl, J.R. Dadamio, T.G. Owano and C.H. Kruger, Chem. Phys. Lett. 234 (1995) 269

[8] R.T. Jongma, M.G.H. Boogaarts, I. Holleman and G. Meijer, Rev. Sci. Instrum. 66 (1995) 2821.

[9] D. Romanini and K.K. Lehmann, J. Chem. Phys. 99 (1993) 6287; 102 (1995) 633.

[10] D.L. Huestis, R.A. Copeland, K. Knutsen, T.G. Slanger, R.T. Jongma, M.G.H. Boogaarts and G. Meijer, Can. J. Phys. 72 (1994) 1109

[11] J.J. Scherer, D. Voelkel, D.J. Rakestraw, J.B.P.C. Collier, R.J. Saykally and A. O'Keefe, Chem. Phys. Lett. 245 (1995) 273.

[12] R. Engeln and G. Meijer, Rev. Sci. Instrum., in press.

[13] J.M. Herbelin, J.A. McKay, M.A.Kwok, R.H. Ueunten, D.S. Urevig, D.J. Spencer and D.J. Benard, App. Opt. 19 (1980) 144.

[14] D.Z. Anderson, J.C. Frisch and C.S. Masser, App. Opt. 23 (1984) 1238.

[15] F. Duschinsky, Z. Phys. 81 (1933) 7.

[16] J.B. Birks and W.A. Little, Proc. Phys. Soc. 66 (1953) 61.

[17] E.A. Bailey Jr. and G.K. Rollefson, J. Chem. Phys. 21 (1953) 1315.

[18] J.R. Lakowics and K.W. Berndt, Rev. Sci. Instrum. 62 (1991) 1727.

[19] S. Gerstenkom and P. Luc, Atlas du spectroscopie d'absorption de la molecule d'iode (CNRS, Paris, 1978). 